\title{
Distributed Particle Filtering for Large Scale Dynamical Systems
}

\author{
Arash Mohammadi and Amir Asif \\ Department of Computer Science and Engineering \\ York University, Toronto, ON, Canada M3J 1P3 \\ Email:\{marash,asif\}@cse.yorku.ca
}

\begin{abstract}
The paper proposes a near-optimal, distributed implementation of the particle filter for large scale dynamical systems with sparse measurements, as in wireless sensor networks (WSN) and power distribution systems. Compared to the centralized approach, the distributed implementation of the particle filter is computationally efficient and provides considerable transmission bandwidth savings due to a significant decrease in information exchange between the neighbouring nodes. In our test simulations, the performance of the distributed implementation is almost indistinguishable from the centralized particle filter.
\end{abstract}

Index Terms - Particle filtering, Distributed Implementation, Bayesian Estimation, Kalman filtering, Large Scale Dynamical Systems.

\section{INTRODUCTION}

Estimating the state variables of a dynamical system from noisy measurements is of significance in many practical situations arising in fields as diverse as physics, biology, engineering, chemistry, meteorology, and pharmacology, to name a few. Dynamical systems are usually modeled with a state space representation and based on this representation, a Bayesian estimator that maximizes the posterior expected value of a utility function is developed to estimate the values of the state variables. For linear systems corrupted with Gaussian excitation and observation noise, the Kalman filter provides the optimal approach. Nonlinear systems with nongaussian excitation implies that in general no analytic solution exists. Consequently, the direct Kalman filter cannot be used and one has to rely on numerical solutions. In such cases, the particle filters [1] are frequently used to estimate Bayesian models and are the sequential ('on-line') analogue of the extended Kalman filter (EKF) and the Unscented Kalman filter (UKF) with the advantage that, with sufficient samples, they approach the Bayesian optimal estimate. Therefore, the particle filter can be made more accurate than either the EKF or the UKF.

A second problem that arises in dynamical systems is the large dimensionality of the estimation problem. Centralized implementation of the particle filters, based on the conventional filtering approach [1], is not feasible in modeling large scale dynamical system. In the centralized approach, each node communicates its observation to a central node, referred to as the fusion center [1], thus, the fusion center is responsible for the overall computation and estimation. Although

This work was supported in part by the Natural Science and Engineering Research Council (NSERC), Canada under Grant No. 228415-2010. the centralized approach is optimal, it is neither robust nor scalable to complex large-scale dynamical systems where the measurement nodes are distributed across a large geographical area. Extensive computation is required to implement the centralized procedure because of the high-dimensionality of large-scale system. Also, a huge amount of information needs to be communicated during each time lag, which is itself a big problem and, among other issues, adds latency to the estimation mechanism.

Although several different approaches have been proposed in the signal processing literature to cope with drawbacks of the centralized particle filter method [2], [3], but researches on the decentralized or distributed particle filter is somewhat limited. Almost all existing distributed particle filters approaches [4], [5] are developed for monitoring low dimensional dynamical systems. These approaches duplicate a particle filter and estimate the entire (not a subset of) state vector at each observation node. Such a scheme is only practical when the dimension of the state vector is small. An alternate approach is used in [6], where a distributed particle filter is developed assuming that the local observations at neighbouring nodes are independent. Such an assumption is over-simplifying and nonpractical in most engineering applications.

In this paper, a near-optimal distributed implementation of the particle filter for large scale dynamical systems with sparse measurements is presented. The distributed approach offers two advantages over the centralized approach. First, unlike the centralized approach, which is computationally intense and practically infeasible for systems with dimensions of large magnitudes, the distributed implementation is computationally feasible and provides significant savings. Second, the centralized approach requires a large amount of information exchange between the observation nodes at each iteration. This adds latency to the system. The distributed approach has a lower latency since it requires reduced number of information transfers between neighbouring nodes. Applications of interest include power constrained wireless sensor networks (WSN) [7] such as the ones used to observe physical processes that are characterized by: (i) non-linear dynamical models; (ii) sparse, highly localized observation sets at the nodes, and; (iii) Non-Gaussian processes. The observation model is, therefore, sparse due to the large dimensionality of the observation field and local nature of the observation transducers. By selecting 
a subset of the state vector at each node, a near-optimal, distributed implementation of the particle filter is proposed that computes the marginal posterior densities for the localized filters at each node. Each localized filter predict and estimate a subset of states with the overall result seen almost the same as the optimal result obtained from the centralized implementation of the particle filter.

The paper is organized as follows. In section II, the centralized particle filter is reviewed. The proposed distributed implementation of the particle filter is presented in Section III. Section IV provides quantitative comparison between the centeralized and distributed implementations of the particle filter by comparing their mean square errors (MSE) for an example simulation. Finally, Section V concludes the paper.

\section{Particle Filter: Centralized Implementation}

The sequential importance sampling (SIS) algorithm is a Monte Carlo (MC) method [8] that forms the basis for most sequential MC filters developed in the last decade [1]. The sequential MC (SMC) approach is also known as the bootstrap filtering, condensation algorithm, and particle filter [1]. It is a suboptimal technique for implementing a recursive Bayesian filter by MC simulations. The key idea in particle filtering is to represent the required posterior density function (pdf) by a set of random samples with associated weights and to compute estimates based on these samples and weights. As the number of samples increase, this $\mathrm{MC}$ characterization becomes an equivalent representation to the usual functional description of the posterior pdf and the SMC filter approaches the optimal Bayesian estimate.

Dynamical systems are usually modeled with a state-space set of models, which involves a process equation and an observation equation as follows

$$
\text { Process Equation: } \quad X_{k}=F\left(X_{k-1}, U_{k-1}, V_{k-1}\right)
$$

Observation Equation: $Z_{k}=G\left(X_{k}, W_{k}\right)$,

where $X$ is a vector composed of the states of dynamical system. Similarly, $Z$ is the observation vector and $U$ the external input (also referred to as the forcing term) to the system. Unlike the Kalman filter, functions $F$ and $G$ can possibly be nonlinear and the noise vectors $\{V, W\}$ are not restricted to have Gaussian distributions.

As discussed above, the particle filter uses a set of 'particles' and associated weighs to estimate the system state $X$. Let $\left\{x_{k}^{i}, w_{k}^{i}\right\}_{i=1}^{N}$ denote a set that characterizes the posterior pdf $p\left(x_{1: k} \mid z_{1: k}\right)$ at time iteration $k$, where $x_{k}^{i}$, for $i=0, \ldots, N$, is a set of support points with associated weights $w_{k}^{i}$, for $i=$ $0, \ldots, N$. The weights are normalized such that $\sum_{i} w_{k}^{i}=1$. The posterior pdf is then approximated as follows

$$
p\left(X_{0: k} \mid Z_{1: k}\right) \approx \sum_{i=1}^{N_{s}} w_{k}^{i} \delta\left(X_{0: k}-X_{0: k}^{i}\right),
$$

where $\delta(\cdot)$ denotes the delta function. The weights are chosen based on the principle of importance sampling explained next. Suppose $p(x)$ approximated by $\pi(x)$ (we use the notation $p(x) \propto \pi(x)$ to represent this approximation) is a pdf from which it is difficult to draw samples but for which $\pi(x)$ is simple to work with and can be evaluated. Also, $x^{i} \sim q(x)$, for $i=0, \ldots, N$, are samples that are easily generated from a proposal density called an importance density. Then, a weighted approximation [1] to the probability density function is given by the multiplication of particles and weights as follows

$$
p(X) \approx \sum_{i=1}^{N_{s}} w^{i} \delta\left(X-X^{i}\right)
$$

where

$$
W^{i} \propto \frac{\pi\left(X^{i}\right)}{q\left(X^{i}\right)},
$$

is the normalized weight of the $i^{t} h$ particle. If the samples were drawn from an importance density $q\left(X_{0: k}^{i} \mid Z_{1: k}\right)$, then the weights in Eq. (5) are defined as

$$
W_{k}^{i} \approx \frac{p\left(X_{0: k}^{i} \mid Z_{1: k}\right)}{q\left(X_{0: k}^{i} \mid Z_{1: k}\right)} .
$$

In the sequential case, we have samples (particles) at each step that approximate the posterior density at last iteration $p\left(X_{0: k-1}^{i} \mid Z_{1: k-1}\right)$ and want to approximate $p\left(X_{0: k}^{i} \mid Z_{1: k}\right)$ with a new set of particles. If we can factorize the importance density as

$$
q\left(X_{0: k} \mid Z_{1: k}\right)=q\left(X_{k} \mid Z_{1: k}, X_{0: k}\right) q\left(X_{0: k} \mid Z_{1: k}\right),
$$

then it is possible to obtain samples $X_{0: k}^{i} \sim q\left(X_{0: k} \mid Z_{1: k}\right)$ by augmenting the new set of samples for the current state $X_{k}^{i} \sim q\left(X_{k} \mid Z_{1: k}, X_{0: k-1}\right)$ to the existing samples $X_{0: k-1}^{i} \sim$ $q\left(X_{0: k-1} \mid Z_{1: k-1}\right)$. The next step is then to update the weights. This is accomplished by expressing $p\left(X_{0: k}^{i} \mid Z_{1: k}\right)$ as

$$
p\left(X_{0: k} \mid Z_{1: k}\right) \propto p\left(Z_{k} \mid X_{k}\right) p\left(X_{k} \mid X_{k-1}\right) p\left(X_{0: k-1} \mid Z_{1: k}\right) .
$$

and substituting Eqs. (7) and (8) in Eq. (5). Simplifying the resulting expression gives us the following equation for sequentially updating the weights

$$
W_{k}^{i} \propto W_{k-1}^{i} \frac{p\left(Z_{k} \mid X_{k}^{i}\right) p\left(X_{k} \mid X_{k-1}^{i}\right)}{q\left(X_{k}^{i} \mid X_{0: k-1}^{i}, Z_{1: k}\right)},
$$

Furthermore, if $q\left(X_{k} \mid X_{0: k-1}, Z_{1: k}\right)=q\left(X_{k} \mid X_{k-1}, Z_{k}\right)$, then the importance density is only dependent on $X_{k-1}$ and $Z_{k}$. This is particularly useful in practice when only a filtered estimate of $p\left(X_{k} \mid Z_{1: k}\right)$ is required at each iteration.

\section{Particle Filtering: Distributed IMPLEMENTATION}

In this paper, we propose a distributed particle filtering approach for large scale dynamical system and sparse observations. In this section, the model reduction of dynamical systems is described first and then the proposed distributed implementation of the particle filter based on the reduced model and sparse observation is introduced. 


\section{A. Modeling large-scale dynamical systems}

In practice, for example in large scale power systems or WSN, observations of each node only depends on a subset of states and not on all of the states. Consequently the observation equation can be represented by a set of equations (one for each node) involving a subset of states as

$$
\begin{aligned}
Z_{k}^{(1)} & =F\left(X_{k}^{(1)}, W_{k}^{1}\right) \\
Z_{k}^{(2)} & =F\left(X_{k}^{(2)}, W_{k}^{2}\right) \\
& \vdots \\
Z_{k}^{\left(N_{s}\right)} & =F\left(X_{k}^{\left(N_{s}\right)}, W_{k}^{N_{s}}\right),
\end{aligned}
$$

where $N_{s}$ is the total number of observation nodes in the system, $X^{l}$, for $l=1,2, \cdots, N_{s}$, are subsets of states observed at each node and $Z^{l}$, for $l=1,2, \cdots, N_{s}$ are observations made at each node. Note that the reduced observation models in Eq. (10)-(12) are coupled because of shared states in the reduced state vectors $X^{i}$ and $X^{j}$, for $1 \leq i, j \leq N_{s}$. One way to reduce the process model is based on the individual states observed at each node as modeled in Eq. (10). This leads to the distributed state model

$$
\begin{aligned}
X_{k}^{(1)} & =F\left(X_{k-1}^{(1)}, D_{k-1}^{(1)}, v_{k-1}^{1}\right) \\
X_{k}^{(2)} & =F\left(X_{k-1}^{(2)}, D_{k-1}^{(2)}, v_{k-1}^{2}\right) \\
& \vdots \\
X_{k}^{\left(N_{s}\right)} & =F\left(X_{k-1}^{\left(N_{s}\right)}, D_{k-1}^{\left(N_{s}\right)}, v_{k-1}^{N_{s}}\right),
\end{aligned}
$$

where, $D^{(l)}$, for $l=1,2, \cdots, N_{s}$, is a subset of states not included in $X^{l}$ but required due to the process model, which may be thought of as internal inputs to each reduced state equation. These external inputs force the system to be coupled and eliminate any additional constraints on the system. The shared states in the process model also indicate that the reduced process model is not decoupled.

An alternate approach for deriving the distributed state and observartion models is discussed next. In many practical systems, the nodes have their own state vector. This means that instead of reducing models based on observation equation and defining the reduced state vectors based on the observation equation, we can define the reduced model based on the system by considering the interconnections of the nodes. For example, in power systems, each generator node has its own state vector consisting of the angular velocity of the generator, transient internal voltage of its armature, and the rotor angle with respect to the machine terminals coupling the system. For such a system, the state variables observed are the active and reactive power components of the terminal, which depend on the state vector of the same node and some states from the neighbouring nodes. In such cases, the model reduction is based on the process model.

\section{B. Distributed recursive Bayesian filter}

Based on the distributed models discussed in the last section, we now introduce the distributed implementation of the particle filter that is localized for each node. The local filters for the nodes estimate a subset of the sates. A reduced number of information transfers of the values of the shared states is still required but the overall transmissions are far less than those required with the centralized particle filter where information on all states need to be communicated. In the distributed implementation, the posterior densities of the reduced models are estimated at each observing node. The posterior densities of each reduced state model are obtained from the marginal distributions

$$
p\left(X^{l} \mid Z\right)=\int_{j \neq l} p(X \mid Z) d X_{j}, \quad \text { for } l, j=1: N,
$$

where $X^{l}$ is a subset of states being filtered at the $l^{\text {th }}$ observation node. The posterior probability $p\left(X^{l} \mid Z\right)$ is a marginal distribution of the global posterior density. By maintaining a filter based on this posterior probability, we can marginally estimate a subset of states locally. Based on the observation model for each sensor, we rewrite this posterior probability as

$$
p\left(X^{l} \mid Z\right)=p\left(X^{l} \mid Z^{(l)}, Z^{\left(S_{l}\right)}\right), \quad \text { for } l, j=1: N,
$$

where $S_{l}$ is a subset of observation nodes that has some shared states with the $l^{\text {th }}$ observation node. In the following discussion, the goal is to compute this posterior proabbaility locally. Without loss of generality and for convenience of representation, the marginal distribution is rewritten as

$$
p\left(X^{l} \mid Z\right)=p\left(X^{l} \mid Z^{(l)}, Z^{(l+1)}\right), \quad \text { for } 1, j=1: N,
$$

where for simplicity it is assumed that only the $\left(l+1^{\text {th }}\right.$ observation node has shared states with the $l^{\text {th }}$ observation node. Using the Baye's rule, the expressions for the posterior densities of $l^{\text {th }}$ node simplify to

$$
\begin{aligned}
p\left(X_{1: k}^{(l)} \mid Z_{1: k}\right) & =\frac{1}{c} p\left(X_{1: k}^{(l)} \mid Z_{(l)}^{1: k-1}, Z_{(l)}^{k}, Z_{(l+1)}^{1: k-1}, Z_{(l+1)}^{k}\right) \\
& =\frac{1}{c} p\left(Z_{k}^{(l)}, Z_{k}^{(l+1)} \mid X_{1: k}^{(l)}, Z_{1: k-1}^{(l)}, Z_{1: k-1}^{(l+1)}\right) \\
& \times p\left(X_{1: k}^{(l)} \mid Z_{1: k-1}^{(l)}, Z_{1: k-1}^{(l+1)}\right)
\end{aligned}
$$

where $c$ is a normalization factor. The first term on the right hand side of Eq.(19) can be written as

$$
\begin{aligned}
& p\left(Z_{(l)}^{k}, Z_{(l+1)}^{k} \mid X_{1: k}^{(l)}, Z_{(l)}^{1: k-1}, Z_{(l+1)}^{1: k-1}\right)= \\
& p\left(Z_{k}^{(l)}, Z_{k}^{(l+1)} \mid X_{1: k}^{(l)}\right) p\left(Z_{k}^{(l+1)} \mid X_{1: k}^{(l)}, Z_{k}^{(l)}\right) p\left(Z_{k}^{(l)} \mid X_{1: k}^{(l)}\right),
\end{aligned}
$$

which is true if we assume that observations are not time correlated, i.e., the observation made at each iteration only depends on the state vector and not on any pervious observations. This marginal posterior density depends not only on the local likelihood and also on the likelihood of neighbouring nodes with which it share some sates. The second part of Eq. (19) also requires some attention. After a few manipulations, we express it as

$$
\begin{array}{r}
p\left(X_{1: k}^{(l)} \mid Z_{1: k-1}^{(l)}, Z_{1: k-1}^{(l+1)}\right)=p\left(X_{1: k-1}^{(l)}, X_{k}^{(l)} \mid Z_{1: k-1}^{(l)}, Z_{1: k-1}^{(l+1)}\right) \\
=p\left(X_{k}^{(l)} \mid X_{1: k-1}^{(l)}\right) p\left(X_{1: k-1}^{(l)} \mid Z_{1: k-1}^{(l)}, Z_{1: k-1}^{(l+1)}\right)
\end{array}
$$




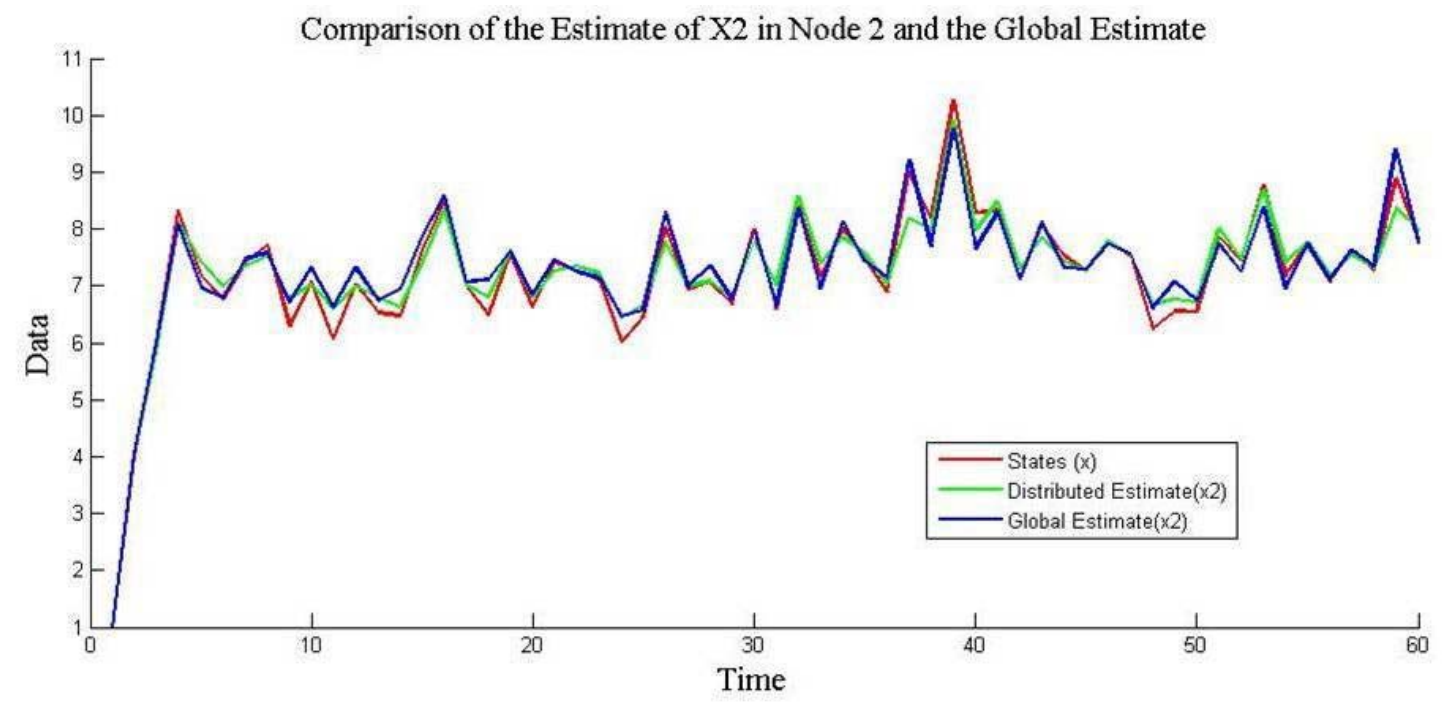

Fig. 1. Comparison of state $x_{2}$ estimated localy at node 2 using the distributed implementation versus the estimate obtained from the centralized implementation. The true value of the state is shown in red.

Eq. (21) relates the local posterior density to the posterior density at the previous iteration. By substituting Eq. (21) and Eq. (20) in Eq. (5), the expression for updating weights is computed as follows

$$
\begin{aligned}
W_{k}^{i,(l)} & \propto W_{k-1}^{i,(l)} \times \\
& \frac{p\left(Z_{k}^{(l+1)} \mid X_{1: k}^{(l)}, Z_{k}^{(l)}\right) p\left(Z_{k}^{(l)} \mid X_{1: k}^{(l)}\right) p\left(X_{k} \mid X_{k-1}^{i}\right)}{q\left(X_{k}^{i} \mid X_{0: k-1}^{i} Z_{1: k}\right)} .
\end{aligned}
$$

\section{EXPERIMENTAL RESULTS}

For testing the performance of the proposed distributed implementation of the particle filter, a power distribution grid with five states and three observation nodes is simulated. The overall state equation is given by

$$
\begin{aligned}
{\left[\begin{array}{l}
x_{1}(k+1) \\
x_{2}(k+1) \\
x_{3}(k+1) \\
x_{4}(k+1) \\
x_{5}(k+1)
\end{array}\right]=} & {\left[\begin{array}{l}
1+\sin \left(.04 \pi x_{1}(k)\right)+x_{2}(k) \\
1+\sin \left(.04 \pi x_{2}(k)\right)+x_{4}(k) \\
1+\sin \left(.04 \pi x_{1}(k)\right)+x_{3}(k) \\
1+\sin \left(.04 \pi x_{4}(k)\right)+x_{5}(k) \\
1+\sin \left(.04 \pi x_{5}(k)\right)+x_{4}(k)
\end{array}\right] } \\
& {\left[\begin{array}{l}
v_{1}(k+1) \\
v_{2}(k+1) \\
v_{3}(k+1) \\
v_{4}(k+1) \\
v_{5}(k+1)
\end{array}\right], }
\end{aligned}
$$

where the forcing terms $v_{i}(k)$ have a Gamma distribution. The observation model composed of 3 sensing nodes is given by

$$
\left[\begin{array}{c}
y_{1}(k) \\
y_{2}(k) \\
y_{3}(k)
\end{array}\right]=\left[\begin{array}{c}
x_{1}(k)+x_{2}(k)+x_{3}(k) \\
x_{2}(k)+x_{3}(k)+x_{4}(k) \\
x_{4}(k)+x_{5}(k)
\end{array}\right]+\left[\begin{array}{c}
w_{1}(k) \\
w_{2}(k) \\
w_{3}(k)
\end{array}\right]
$$

where the observation noise $w_{i}(k)$ is assumed Gaussian. Both direct and distributed implementations of the particle filter are implemented separately. For the distributed implementation, the local filter on the first node follows states $x_{1}, x_{2}$ and $x_{3}$, a subset of the state vector. Similarly, the second node only estimates states $x_{2}, x_{3}$ and $x_{4}$. Finally, the third filter estimates states $x_{4}$ and $x_{5}$. Because the system is coupled, some information is required to be transmitted between the neighbour sensors. In our experiments, for example, the local filter at the first sensor requires information on state $x_{4}$. The information that is shared on state $x_{4}$ its estimate instead of the particles. Similarly, for the other local filters at the remaining nodes.

Fig. (1) provides a comparison between the estimate of $x_{2}$ obtained from the second sensor of the distributed implementation versus its estimate obtained from the centralized approach. The result indicates that the estimates from the distributed and centralized implementation are fairly close. During some iterations, it is observed that the estimate from the distributed implementation is better than the one obtained from the centralized implementation. The point is that the particle filter is itself a suboptimal estimator. Due to the randomness of the particles, the results are not expected to be exactly the same. In order to further compare the proposed distributed method with the centralized method, mean square errors (MSE) averaged over the entire run is compared in Table (1), which illustrated that the average MSE for each state is close to the global estimate. Therefore, we conclude that the proposed distributed particle filter is a near-optimal implementation of the centralized particle filter.

\section{SUMMARY}

In this paper, a near-optimal, distributed implementation of the particle filter for large scale dynamical systems with sparse measurements is proposed. The motivation comes from signal processing applications in wireless sensor networks (WSN) and power transmission systems. For such systems, the number of states to be estimated is large and each node can only observe a subset of the states. Latency is a critical issue as 
TABLE I

DISTRIBUTED IMPLEMENTATION VERSUS CENTRALIZED

IMPLEMENTATION FOR THE PARTICLE FILTER: COMPARISON OF THE MEAN SQUARE ERRORS (MSE) FOR THE TWO

IMPLEMENTATIONS AVERAGED OVER THE ENTIRE RUN.

\begin{tabular}{|c||c||c|c|c|}
\hline \multicolumn{1}{|c||}{} & \multirow{2}{*}{$\begin{array}{c}\text { Centralized } \\
\text { Implementation }\end{array}$} & \multicolumn{3}{c|}{ Distributed Implementation } \\
\cline { 3 - 5 } & & Node 1 & Node 2 & Node 3 \\
\hline \hline$x_{1}$ & 0.0085 & 0.0034 & - & - \\
\hline$x_{2}$ & 0.0026 & 0.0028 & 0.0024 & - \\
\hline$x_{3}$ & 0.0032 & 0.0040 & 0.0024 & - \\
\hline$x_{4}$ & 0.0031 & - & 0.0032 & 0.0026 \\
\hline$x_{5}$ & 0.0029 & - & - & 0.0031 \\
\hline \hline
\end{tabular}

real-time estimates for the states are required to make urgent decisions. Compared to the centralized approach, the proposed distributed implementation of the particle filter is computationally feasible (provides significant computational savings) and offers considerable transmission bandwidth savings due to a significant decrease in information exchange between the neighbouring WSN nodes. Reduction in information exchange improves the latency of the system. In the test simulations included in the paper, the performance of the distributed implementation is indistinguishable from the centralized particle filter.

\section{REFERENCES}

[1] N. Gordon M. Sanjeev, S. Maskell and T. Clapp, "A tutorial on particle filters for online nonlinear/non-gaussian bayesian tracking," IEEE Trans. on Sig. Proc., vol. 50, pp. 174-187, 2002.

[2] X. Sheng and Y. H. Hu, "Sequential acoustic energy based source localization using particle filter in a distributed sensor network," in ICASSP 2004, III, 2004, pp. 972-975.

[3] X. Sheng and Y-H. Hu, "Distributed particle filter with gmm approximation for multiple target localization and tracking in wireless sensor network," in IEEE/ACM Int. Symp. IPSN, Los Angeles, 2005.

[4] G. Gordon M. Rosencrantz and S. Thrun, "Decentralized sensor fusion with distributed particle filters," in Conf. Uncertainty in Artificial Intelligence, Mexico, 2003.

[5] Y. H. Hu and X. Sheng, "Dynamic sensor self-organization for distributive moving target tracking," Journal of Signal Processing Systems, vol. 51, no. 2, pp. 161-171, May 2008.

[6] M.J. Coates, "Distributed particle filtering for sensor networks," in IEEE/ACM Int. Symp. IPSN, Berkeley, 2004.

[7] X. Sheng and Y. H. Hu, "Distributed Particle Filters for Wireless Sensor Network Target Tracking", in ICASSP 2005, 2005.

[8] J. Liu and R. Chen, "Sequential monte carlo methods for dynamic systems," Journal of the American Statistical Association, vol. 93, pp. 1032-1044, 1998. 